\title{
Philosophiques
}

\section{Wittgenstein et la preuve mathématique comme vérifacteur}

\section{Mathieu Marion}

Volume 38, numéro 1, printemps 2011

Les conférences Hugues Leblanc 2010

URI : https://id.erudit.org/iderudit/1005720ar

DOI : https://doi.org/10.7202/1005720ar

Aller au sommaire du numéro

Éditeur(s)

Société de philosophie du Québec

ISSN

0316-2923 (imprimé)

1492-1391 (numérique)

Découvrir la revue

Citer cet article

Marion, M. (2011). Wittgenstein et la preuve mathématique comme vérifacteur. Philosophiques, 38(1), 137-156. https://doi.org/10.7202/1005720ar

\section{Résumé de l'article}

Dans ce texte, je pars de l'analyse intuitionniste de la vérité mathématique, « $A$ est vrai si et seulement s'il existe une preuve de $A$ » comme cas particulier de l'analyse de la vérité en termes de " vérifacteur ", et je montre pourquoi Wittgenstein partageait celle-ci avec les intuitionnistes. Cependant, la notion de preuve à l'oeuvre dans cette analyse est, selon l'intuitionnisme, celle de la " preuve-comme-objet », et je montre par la suite, en interprétant son argument sur le caractère " synoptique " des preuves, que Wittgenstein avait plutôt en tête une conception de la " preuve-comme-trace ". 


\title{
Wittgenstein et la preuve mathématique comme vérifacteur'
}

\author{
MATHIEU MARION \\ Université du Québec à Montréal
}

\begin{abstract}
RÉSUMÉ. - Dans ce texte, je pars de l'analyse intuitionniste de la vérité mathématique, «A est vrai si et seulement s'il existe une preuve de $A \gg$ comme cas particulier de l'analyse de la vérité en termes de «vérifacteur», et je montre pourquoi Wittgenstein partageait celle-ci avec les intuitionnistes. Cependant, la notion de preuve à l'œuvre dans cette analyse est, selon l'intuitionnisme, celle de la «preuve-comme-objet», et je montre par la suite, en interprétant son argument sur le caractère «synoptique» des preuves, que Wittgenstein avait plutôt en tête une conception de la «preuve-comme-trace».
\end{abstract}

ABSTRACT. - In this paper, I start with the intutionist analysis of mathematical truth, «A is true if and only if there exists a proof of $A »$, as a particular case of the analysis of truth in terms of «truth-makers », and I show why Wittgenstein shared it with the intuitionists. However, the notion of proof at work in this analysis is, according to intuitionism, that of «proof-as-object», and I then show, with an interpretation of his argument on the «surveyability» of proofs, that, instead, Wittgenstein had in mind a notion of «proof-as-trace».

\section{La preuve intuitionniste comme «vérifacteur »: acte, trace ou objet?}

Dans «Truth-Makers », Kevin Mulligan, Peter Simons et Barry Smith ont proposé une analyse générale de la notion de vérité en termes de «truth-makers» ou "vérifacteurs", devenue depuis centrale en philosophie analytique ${ }^{2}$ :

(1) $A$ est vrai si et seulement s'il existe un vérifacteur pour $A^{3}$.

Göran Sundholm a proposé dans «Existence, Proof and TruthMaking: A Perspective on the Intuitionistic Conception of Truth» d'inclure

1. Dans ce texte, les références au Tractatus logico-philosophicus sont au numéro de paragraphe et non au numéro de page de la traduction de Gilles-Gaston Granger (Wittgenstein, 1993). Dans ce cas comme dans celui des autres traductions françaises de Wittgenstein, je me permets de les modifier silencieusement au besoin.

2. Mulligan, Simons \& Smith, 1984. Pour des discussions récentes, voir Beebee \& Dodd, (2005), Lowe \& Rami (2009) ou Monnoyer (2007). Bien sûr, l'analyse de la vérité en termes de vérifacteurs est bien plus ancienne, plusieurs sources étant déjà répertoriées dans Mulligan, Simons \& Smith (1984), à commencer par le Tractatus logico-philosophicus de Wittgenstein; tout récemment, Mulligan a montré qu'en 1921, l'année de la publication du Tractatus, des philosophes aussi distants que J. E. M. McTaggart et Alexander Pfänder ont aussi proposé des analyses en termes de "vérifaction» (truth-making) (Mulligan, 2009).

3. Une version plus exacte de ce principe serait: «Pour tout $x, x$ est vrai si et seulement s'il existe un $y$ tel que $y$ rend $x$ vrai". 
comme cas particulier de cette analyse générale l'analyse intuitionniste de la vérité mathématique ${ }^{4}$ :

(2) A est vrai si et seulement s'il existe une preuve de $A$.

Les éléments de cette suggestion étaient déjà présents dans les écrits de Sir Michael Dummett, bien connu pour avoir défendu la thèse $(2)^{5}$, tandis qu'il admet par ailleurs dans «What is a Theory of Meaning ? II " un «principe $C$ » proche de (1), qu'on pourrait formuler comme suit:

(3) Si $A$ est vrai, alors il doit y avoir quelque chose qui rend $A$ vrai ${ }^{6}$.

Cependant, Dummett n'a jamais développé une analyse de la notion intuitionniste de intuitionniste en termes de vérifacteurs. Dans ce texte, mon but sera, modestement, celui d'un historien de la philosophie, pour qui un développement contemporain comme celui-ci peut aider à mieux comprendre les philosophes du passé. Cela va déjà de soi dans le cas de (1) et de (2), puisque ces analyses ont des sources historiques évidentes, mais j'aimerais illustrer la valeur de cette analyse en l'appliquant à un domaine plutôt inusité, la philosophie des mathématiques de Wittgenstein.

Dans une analyse du type de (1) ou de (2), il faut bien sûr préciser la nature des «truth-bearers » ou "porteurs de vérité », c'est-à-dire la nature de $A$, et il faut expliquer ce que peut bien être un vérifacteur de $A$. Il faut aussi mettre au clair la notion d'existence propre à cette catégorie. Il reste en outre à préciser dans (2) ce qu'on y entend par «preuve». Dans son texte, Sundholm fait appel, en plus de Dummett, à Ludwig Wittgenstein, Arend Heyting - dont il pointe du doigt les sources chez Brouwer, Husserl et Oskar Becker -, A. N. Kolmogorov, Hermann Weyl et Per Martin-Löf. Mais le cœur de son analyse fait essentiellement appel à un article célèbre de Heyting, où celui-ci propose que les porteurs de vérité $A$ dans (2) soient les "propositions » mathématiques ${ }^{7}$. Utilisant un vocabulaire emprunté de la phénoménologie, Heyting distingue entre une "proposition» (Aussage) et son «assertion» (Satz): une proposition mathématique telle que «La constante d'Euler est rationnelle» exprime une «attente»(Erwartung), qui est de trouver deux entiers $a, b$ tels que $C=a / b$, tandis que l'assertion $(S a t z)$ correspond à "l'affirmation de la proposition », c'est-à-dire à la «réalisation » (Erfüllung) de cette "attente» par la «détermination empirique d'un fait» (die Feststellung einer empirischen Tatsache), à savoir l'existence d'une

4. Sundholm (1994a); voir aussi Sundholm (1993, 59), et (1994b, 294).

5. Énoncée, par exemple, dans Dummett (2000, 4).

6. Il ne s'agit pas de la formulation exacte de Dummett: «Si un énoncé est vrai, il doit y avoir quelque chose en vertu de quoi celui-ci est vrai» (Dummett, 1993, 52).

7. Heyting, 1931, 113. Sundholm revient souvent sur son analyse des textes de Heyting, par exemple dans Sundholm (1983, 156-161) (1993, 55 et 59-60), et (1994b, 298-299). 
preuve qui fournit, pour la constante d'Euler $C$, les deux entiers $a, b$ tels que $C=a / b^{8}$. Cette assertion (le théorème démontré) est de la forme :

(4) La proposition «La constante d'Euler est rationnelle» est vraie.

Une analyse du même type a été proposée en 1932 par Kolmogorov pour qui une proposition mathématique $a$ pose un "problème " (Aufgabe) et correspond à sa "solution» (Lösung $)^{9}$. Ces analyses sont en accord avec l'intuitionnisme de Brouwer $^{10}$, et elles sont à la base de ce qu'on appelle aujourd'hui la sémantique «BHK», pour "Brouwer-Heyting-Kolmogorov».

Comme le remarque Sundholm, à la suite cette fois-ci d'une suggestion de Martin-Löf ${ }^{11}$, une preuve est, dans ce contexte, à la fois subjective et objective $^{12}$. En effet, en tant que démonstration, la preuve peut être vue comme l'acte ou les actes par lesquels le mathématicien réussit à connaître la vérité d'une proposition mathématique $p$. Une telle démonstration a pour objet le théorème prouvé selon lequel la proposition $p$ est vraie. Une fois effectués, ces actes n'existent plus, mais le mathématicien peut laisser des traces, objectives cette fois-ci, qu'on retrouve dans les livres de mathématique et dont les autres mathématiciens se servent pour effectuer pour euxmêmes les mêmes actes subjectifs, c'est-à-dire pour refaire la preuve du théorème selon lequel la proposition $p$ est vraie. On doit donc distinguer entre deux notions :

(i) La preuve-comme-acte ou démonstration,

(ii) La preuve-comme-trace.

Sundholm montre cependant que la notion de preuve appelée à jouer le rôle de vérifacteur dans l'analyse intuitionniste de la vérité n'est ni l'une, ni l'autre, mais une notion de preuve comme objet mathématique, pour laquelle Brouwer avait introduit l'expression "Beweisführung ", dans sa preuve du théorème de la barre de $1924^{13}$. Pour Brouwer, comme pour Heyting à sa suite:

8. Heyting 1931, 113. La même analyse était déjà présentée dans un texte moins bien connu de 1930, en français: "L'affirmation. Une proposition p, comme, par exemple, «la constante d'Euler est rationnelle", exprime un problème, ou mieux une certaine attente (celle de trouver deux entiers $a$ et $b$ tels que $C=a / b$ ), qui pourra être réalisée ou déçue. [...] Remarquons encore que, en logique classique comme en logique intuitionniste, l'affirmation d'une proposition n'est pas elle-même une proposition, mais la constatation d'un fait " (Heyting, 1930, 958-959).

9. Voir Kolmogorov (1932); Heyting fait déjà allusion à cette analyse dans le passage cité dans la note précédente ainsi que dans Heyting $(1931,114)$.

10. Voir Brouwer (1992) pour le texte fondateur de l'intuitionnisme.

11. Sundholm (1993, 124 n. 15).

12. Ce paragraphe suit de près Sundholm $(1994,121-122)$ et (1998a, 180), auquel mon argument demande que l'on souscrive.

13. Voir son article «Beweis, dass jede volle Funktion gleichmässig stetig ist », reproduit dans Brouwer (1976, 286-290). 
La preuve d'une proposition est une construction mathématique qui peut être traitée elle-même mathématiquement ${ }^{14}$.

En effet, selon l'intuitionniste, c'est-à-dire en accord avec (2), plutôt que (4), on devrait lire:

(5) La construction $C=a / b$ est une preuve de la proposition « La constante d'Euler est rationnelle ",

ou plus généralement

(6) La construction $c$ prouve $A$,

tandis que cette construction $c$ peut elle-même devenir un objet mathématique. On a donc une troisième notion:

(iii) La preuve-comme-objet.

La proposition «La constante d'Euler est rationnelle» exprime donc une "attente» sous la forme d'un objet mathématique satisfaisant certaines conditions, et la vérité de cette proposition sera démontrée par la construction d'une preuve-comme-objet satisfaisant ces conditions; pour Sundholm, la notion intuitionniste de vérifacteur dans (2) est donc celle de preuvecomme-objet ${ }^{15}$.

Dans ce qui suit, j'aimerais simplement montrer que pour Wittgenstein, du moins dans certains de ses textes, la notion intuitionniste de vérifacteur dans (2) serait plutôt celle de preuve-comme-trace, c'est-à-dire que l'idée d'une preuve-comme-objet ne l'effleure pas. En fait, il ne discute pas de ces questions, mais j'aimerais montrer, par l'étude d'un seul exemple, celui de son argument à propos du "caractère synoptique " (Übersichtlichbarkeit, surveyability) des preuves, qu'il présuppose l'idée de preuve-commetrace. Il n'est pas question de décider ici si la bonne notion pour l'intuitionnisme doit être celle de preuve-comme-objet ou non. On pourrait blâmer Wittgenstein pour ne pas avoir entrevu cette notion, pourtant centrale pour la sémantique «BHK», mais il faut cependant noter que, de Kreisel à Sundholm, l'idée d'une "théorie des constructions », telle que suggérée par (6), s'est avérée infructueuse ${ }^{16}$. Toutefois, je dois auparavant montrer que Wittgenstein, qui est pourtant considéré comme un des pionniers de l'analyse de la vérité en termes de vérifacteurs dans le Tractatus logico-philosophicus, a bien soutenu quelque chose comme (2).

14. Heyting $(1931,114)$.

15. Sundholm (1994, 121-122).

16. Il n'y eut en effet guère de progrès depuis Sundholm (1982), qui donne un historique de cette approche. 


\section{Wittgenstein, la logique et les équations de la théorie des nombres}

Selon une lecture largement répandue, la conception de l'inférence mise de l'avant dans le Tractatus logico-philosophicus est à rapprocher de la notion de «conséquence logique» développée de Bolzano à Tarski. Dans cette tradition, on définit l'inférence de $A 1, \ldots, A k$ à $C$ comme étant valide si et seulement si dans tous les «cas» où les prémisses $A 1, \ldots, A k$ sont vraies, la conclusion $C$ est elle aussi vraie. Lorsque (7) est valide, on dit que " $C$ est une conséquence logique de $A 1, \ldots, A k$ », ce qu'on écrit de la façon suivante:

(7) $A 1, \ldots, A k \mid=C$

On peut aussi rendre (7) par

(8) $A 1$ est vrai, ..., Ak est vrai, donc $C$ est vrai.

La validité d'une inférence est ainsi réduite à l'obtention de cette relation de conséquence logique entre propositions ${ }^{17}$. Cette définition se trouve déjà in nuce dans la Wissenschaftslehre de Bolzano et elle a été formulée de façon adéquate pour la première fois par Alfred Tarski en 1937 dans son article "Sur le concept de conséquence logique ", de la manière suivante:

(9) Une proposition $X$ «suit logiquement » d'une classe $K$ de propositions si et seulement si tout modèle de la classe $K$ est aussi un modèle de la proposition $X^{18}$.

La définition que propose Wittgenstein dans le Tractatus logico-philosophicus en 1921 est donc fréquemment perçue comme préfigurant celle de Tarski:

5.12 - En particulier, la vérité d'une proposition « $p$ » suit de la vérité d'une proposition «q» quand tous les fondements de vérité de la seconde sont des fondements de vérité de la première.

5.121 - Les fondements de vérité de l'une sont contenus dans ceux de l'autre: $p$ suit de $q$.

Ce qui fait l'intérêt de Wittgenstein dans le cadre de (1), c'est qu'il postule pour chaque « $A$ est vrai» dans (8) un corrélat ontologique, un «état de choses" (Sachverhalt) comme vérifacteur de $A-$ ce que Bolzano ou Tarski ne font pas.

La définition (7), pourtant tout à fait «standard» de nos jours, se heurte à de nombreuses difficultés. Pour ne prendre qu'un exemple, il faut pouvoir définir ce qu'on entend ci-dessus par "tous les cas", or il existe au moins deux façons différentes de spécifier ce qu'est un "cas», ce qui ouvre

17. Pour plus de détails sur cette réduction de la validité de l'inférence à la conséquence logique en propositions, voir Sundholm (1998a) et (1998b).

18. Tarski $(1974,150)$. 
la porte dans le cadre de cette approche au pluralisme logique ${ }^{19}$. Tarski luimême reconnaissait dans son article "Sur le concept de conséquence logique" que sa définition n'était qu'une la formalisation d'une notion intuitive qu'elle ne parvient pas à capturer parfaitement. La raison en est que les propositions $A 1, \ldots, A k$ dans (7), (8) et (9) doivent être "analytiques " pour que l'inférence soit valide "dans tous les cas ", en ce sens que chacune de ces propositions doit rester vraie "sous toute variation de son vocabulaire non logique» et qu'il n'existe, comme le reconnait Tarski, aucun critère objectif pour séparer le vocabulaire «logique» du vocabulaire «non logique $»^{20}$. Tarski et Quine, lui aussi bien connu pour avoir reconnu ce fait, ont malgré cela tous deux cherché en vain à en fournir un ${ }^{21}$.

Quoi qu'il en soit de ces difficultés, ou encore du fait que cette interprétation de la notion d'inférence dans le Tractatus logico-philosophicus puisse elle-même être controversée ${ }^{22}$, il reste qu'elle ne s'applique pas dans l'esprit de Wittgenstein au domaine qui nous intéresse, à savoir les mathématiques. Avant d'expliquer pourquoi il en est ainsi, il faut simplement noter que la discussion de Wittgenstein ne porte dans son livre que sur la théorie des nombres, jamais sur les mathématiques en général — et ce malgré l'imprécision de ses propres formulations - et qu'il n'y considère jamais la théorie des nombres sous l'angle des preuves, uniquement celui des calculs numériques. En effet, malgré une définition inductive de la suite des nombres naturels, présentée ci-dessous, il ne mentionne même pas les preuves par induction mathématique. Ce n'est qu'après son retour à Cambridge en 1929 qu'il élargira sa conception pour rendre compte des preuves en théorie des nombres.

À l'opposé de Frege et de Russell, qui cherchent à exprimer la théorie des nombres dans un langage logique en donnant une interprétation logique de l'arithmétique de Peano, Wittgenstein développe donc un calcul des équations, proche de ce que nous appellerions aujourd'hui le $\lambda$-calcul, dans un langage, celui des "opérations", clairement distinct du langage logique. Les idées de Wittgenstein peuvent être reconstruites et présentées (en usant

19. Voir Beall et Restall (2006). Le pluralisme logique peut certes être vu comme une bonne chose - c'était déjà l'avis de Carnap - mais la plupart des tenants de cette approche sont des «monistes", et considèrent qu'il n'existe qu'une seule logique, en l'occurrence la logique classique. Mais il ne peuvent pas fermer complètement la porte au pluralisme.

20. Tarski (1974, 152-153).

21. Voir Tarski (1986) et Quine (1980) et $(1986,29)$. La suggestion de Quine, portant sur la taille de la classe de variation ou «substitution salva congruitate», est moins bien connue que celle de Tarski.

22. J'ai donné des raisons de croire que cette interprétation de la notion d'inférence dans le Tractatus logico-philosophicus est erronée dans Marion (2001); voir aussi Granger (1990). Mais cette question n'a pas besoin d'être discutée ici. 
de liberté sur le plan de la notation) autour de l'idée centrale ${ }^{23}$ selon laquelle pour deux termes numériques $t$ et $s$, l'équation:

$$
t=s
$$

tient si et seulement si, dans ce langage des opérations, on a :

$\Omega^{\mid \mathrm{t} t} a=\Omega^{\mid \mathrm{s} \mathrm{l}} a$.

Wittgenstein définit une "série de formes» (Formenreihe) comme étant ordonnée par une "relation interne» (4.1252), c'est-à-dire comme étant « équivalente à l'opération par laquelle un terme de la série est engendré par un autre» (5.232). Avec une "opération", qui, contrairement à une "fonction» dans le langage logique, peut prendre son propre résultat comme base de son application, on peut ainsi progresser de terme à terme dans une «série de forme» $(5,252)$. Si on prend pour exemple « $\Omega$ ' $x$ », le symbole « $\Omega$ » est la variable pour l'opération et il représente, quand il est lié à l'apostrophe, le résultat de l'application de l'opération, tandis que « $x »$ est la variable pour un terme quelconque dans la «série de forme». Si on prend « $a »$ comme base, c'est-à-dire comme n'étant pas le résultat d'une application précédente de « $\Omega$ », l'expression « $\Omega$ ' $a$ » représentera donc le résultat de la première application de l'opération $« \Omega$ ». L'opération peut ensuite être appliquée à nouveau à ce résultat " $\Omega^{\prime} a$ ", et on obtient " $\Omega^{\prime} \Omega^{\prime} a$ ", etc. On obtient ainsi la «série de forme»:

$$
\text { a, } \Omega^{\prime} a, \Omega^{\prime} \Omega^{\prime} a, \Omega^{\prime} \Omega^{\prime} \Omega^{\prime} a, \Omega^{\prime} \Omega^{\prime} \Omega \text { ' } \Omega^{\prime} a, \ldots
$$

Wittgenstein propose au 5.2522 comme "variable" pour ces «séries de forme» :

$$
\left[a, x, \Omega^{\prime} x\right]
$$

Ce n'est certes pas une variable au sens ou nous l'entendons de nos jours, mais l'expression se lit simplement: « $a$ » indique la base, « $x »$ un terme quelconque de la série et " $\Omega$ ' $x$ » le résultat de l'application de l'opération à ce terme pour en produire le successeur dans la série. Il s'agit d'une forme de définition par induction. Les crochets ont pour but d'indiquer qu'on ne peut écrire en dehors de ceux-ci, pour éviter entre autres les problèmes de circularité qu'il a diagnostiqués dans les définitions de Frege et de Russell au 4.1273. Wittgenstein peut donc définir au 6.02 les entiers comme « exposants ", c'est-à-dire en termes de répétition de l'application d'une opération:

$$
\begin{aligned}
& a=\Omega^{0} a \\
& \Omega^{\prime} a=\Omega^{1} a
\end{aligned}
$$

23. Voir Frascolla $(1994,3)$ et Frascolla (1997), ainsi que Marion $(1998,26)$ et Potter (2000, 182). 


$$
\Omega^{\prime} \Omega^{\prime} a=\Omega^{2} a
$$

etc.

En remplaçant les apostrophes par des parenthèses ${ }^{24}$, et avec $s$ pour le successeur dans la "série de formes ", on obtient la définition inductive suivante, qui préfigure celle de Church dans le $\lambda$-calcul:

$$
\begin{aligned}
& \Omega^{0} a={ }_{\text {Def }} a \\
& \Omega^{\text {sn }} a={ }_{\text {Def }} \Omega\left(\Omega^{n} a\right)
\end{aligned}
$$

Suivant la «variable» introduite au 5.2522, il peut donc symboliser au 6.03 la suite des nombres naturels de cette manière:

$$
[0, \xi, \xi+1]
$$

La preuve de prime abord ésotérique de « $2 \times 2=4$ » au 6.241 permet de retrouver les définitions de l'addition et de la multiplication ${ }^{25}$ :

$$
\begin{aligned}
& \Omega^{m+n} a={ }_{\text {Def }} \Omega^{m}\left(\Omega^{n} a\right) \\
& \Omega^{m \times n} a={ }_{\text {Def }}\left(\Omega^{n}\right)^{m} a
\end{aligned}
$$

On se retrouve ainsi avec un système de réécriture, dans le sens de la $\beta$-réduction du $\lambda$-calcul. On peut en effet montrer qu'on peut réécrire l'expression à gauche du signe d'égalité sous la forme à sa droite dans:

$$
\Omega^{2 \times 2} a=\Omega^{4} a
$$

par une série de substitutions, que Wittgenstein appellera après 1929 une "chaîne d'équations" (Gleichungskette), ce qui est consigné de manière abrégée par l'expression « $2 \times 2=4 »$. C'est ce que dit Wittgenstein au 6.24:

6.24 - La méthode dont use la mathématique pour obtenir ses équations est la méthode de substitution.

Les équations en effet expriment la substituabilité de deux expressions, et nous procédons d'un certain nombre d'équations, en substituant, conformément aux équations, des expressions à d'autres.

Comme je l'ai dit, Wittgenstein a donc entre les mains quelque chose qui se rapproche du $\lambda$-calcul. Étant donné le fait qu'il peut donc rendre compte par là des fonctions $\lambda$-définissables, et étant donné la «thèse de Church ", il avait donc entre les mains, même s'il ne l'a pas pleinement réalisé, un calcul d'une portée qui est loin d'être négligeable. Mais il est clair qu'il tenait à ce que ce langage des équations de la théorie des nombres soit tenu à l'écart de celui de la logique. Wittgenstein ne voyait pas le langage des Principia Mathematica comme approprié pour l'expression des équations de

24. Voir Potter $(2000,184)$.

25. Idem. 
la théorie des nombres, et ce non seulement à cause de vices particuliers à la théorie des types ramifiés, comme ceux qu'il relève à propos de l'axiome d'infinité (5.535) et de l'axiome de réductibilité (6.1232-6.1233), mais tout simplement parce que l'appareil de la théorie de la quantification et sa notion logique de prédicat $F(x)$ ne sont pas appropriés. Il suffit pour s'en rendre compte, de prendre l'exemple du principe d'induction mathématique, donné ici dans une forme simplifiée - Frege et Russell donnaient des versions plus complexes au second ordre - avec $s$ pour la fonction de successeur:

$$
(F(0) \& \forall x(F(x) \rightarrow F(s(x))) \rightarrow \forall x F(x),
$$

Si on prend ici $F(x)$ dans toute sa généralité, alors le principe exprime beaucoup plus que la simple égalité entre termes numériques que Wittgenstein cherchait à capturer avec son langage des opérations ${ }^{26}$.

Ces considérations nous permettent de mieux comprendre les remarques de Wittgenstein sur les mathématiques dans son Tractatus logicophilosophicus. Elles ont en effet pour but de montrer que, pour celui-ci, les équations de la théorie des nombres montrent qu'un terme peut être substitué à un autre sans que cela puisse être asserté (6.1263). Car si on pouvait asserter ce qui se montre par ces "chaînes d'équations ", alors on pourrait et devrait exprimer ces équations dans le langage de la logique, en l'occurrence celui des Principia Mathematica, ce dont Wittgenstein ne veut manifestement pas. En proposant un calcul des équations, Wittgenstein peut rendre compte des équations de la théorie des nombres sans faire appel au langage de la logique, il lui suffit donc d'expliquer pourquoi les équations ne doivent pas être comprises comme des assertions. Son argument à cet effet est simple et s'appuie sur la distinction centrale de l'œuvre, celle-là même qui est rejetée par la lecture dominante aujourd'hui, le «New Wittgenstein ${ }^{27}$, à savoir la distinction entre "dire» et "montrer» (4.12-4.1212). Pour Frege, une équation telle que « $2 \times 2=4$ » asserte que les deux membres de l'équation sont en fait deux sens (Sinn) différents qui ont la même signification (Bedeutung), c'est-à-dire que les deux sens réfèrent à un seul et même objet abstrait. En remplacement de cette analyse, Wittgenstein propose de lire les équations comme n'étant pas "propositionnelles"; elles sont plutôt selon lui des "pseudo-propositions» (Scheinsätze) (6.2), qui n'expriment donc aucune «pensée» (6.21) au sens particulier où il entend ce concept (par exemple au paragraphe 4). Selon lui, le signe d'égalité montre que les deux expressions qu'il relie ont la même signification, en ce sens qu'on peut voir que l'une se laisse réécrire en l'autre, comme je l'ai expliqué (les deux expressions sont donc substituables):

26. Voir sur cette question Marion \& Okada (2011).

27. Cette lecture dérive de Diamond (2004). 
6.23 - Si deux expressions sont liées par le signe d'égalité, cela signifie qu'elles peuvent être substituées l'une à l'autre. Mais que cela soit le cas doit se montrer dans les deux expressions elles-mêmes [an den beiden Ausdrücken selbst zeigen]. $[\ldots]$

6.232 - Frege dit que les deux expressions ont la même signification [Bedeutung], mais des sens [Sinn] différents.

Mais l'essentiel dans l'équation est qu'elle n'est pas nécessaire pour montrer que les deux expressions liées par le signe d'égalité ont la même signification, car cela se laisse voir à partir des deux expressions elles-mêmes.

6.2321 - Et que les propositions de la mathématique puissent être démontrées, cela ne veut rien dire d'autre sinon que leur rectitude [Richtigkeit] est percevable [einzusehen ist] sans que ce qu'elles expriment doive être comparé avec les faits pour en établir la rectitude.

La rectitude d'une équation se montre donc et peut donc être reconnue comme telle am Symbol allein (tout comme la tautologie peut l'être, cela étant, selon le 6.113, l'idée clef de toute la philosophie de la logique). Puisque l'identité de signification ne peut pas faire l'objet d'une assertion (6.2322), le signe d'égalité ne tourne pas l'équation en une assertion, il ne réfère donc à rien et ne fait qu'indiquer le point de vue à partir duquel on peut reconnaître que les deux expressions sont substituables:

6.2323 - L'équation ne fait qu'indiquer le point de vue à partir duquel je considère les deux expressions, c'est-à-dire le point de vue de leur égalité de signification [Bedeutungsgleichheit].

Wittgenstein peut donc faire avec cette analyse l'économie des nombres conçus comme objets abstraits à propos desquels les équations de la théorie des nombres seraient censées être des assertions; il rejette donc ce qu'on peut appeler le «platonisme» de Frege. Mais il ne fait pas que cela: étant donné que la logique s'applique aux propositions et que les équations de la théorie des nombres ne sont pas des propositions, la logique ne s'applique donc pas à ces dernières - c'est la conclusion recherchée. En d'autres termes, la notion de conséquence logique enchâssée dans (7) ci-dessus ne s'applique pas aux équations de la théorie des nombres. Voilà pourquoi il ne peut y avoir pour Wittgenstein d'application du principe (1) pour ces équations.

Mais Wittgenstein défendait-il pour autant un principe comme (2) pour les mathématiques? Cette question est délicate, puisqu'une réponse positive ferait de Wittgenstein un constructiviste, et la plupart des interprètes de son œuvre sont convaincus qu'il n'a jamais soutenu une position de ce genre. Je crois pour ma part qu'ils n'ont simplement jamais vraiment réfléchi à ces questions; la plupart des lecteurs de Wittgenstein y cherchent plutôt à confirmer leur propre projet philosophique, plutôt que de simplement chercher à comprendre, et donc reconstruire de la manière la plus 
cohérente possible, ce qu'il a voulu dire. S'il n'y a pas de réalité mathématique qui rend nos énoncés vrais ou faux, que ce soit de manière indépendante ou, selon l'anti-réalisme de Dummett, suivant notre possibilité de les reconnaître comme vrais ou faux, alors Wittgenstein n'a guère d'autre choix que de faire reposer le fardeau de la «vérifaction» sur la preuve mathématique. Ce qui veut dire qu'il ne peut pas accepter un concept réaliste d'existence mathématique et donc qu'il doit rejeter en toute cohérence les preuves qui se contentent (souvent en tant que conclusion d'une reductio ad absurdum utilisant le principe du tiers-exclu critiqué par les intuitionnistes) d'asserter que « $\exists x F(x)$ » tout en n'étant pas en mesure de fournir ce $x$; ce qui est par ailleurs déjà exclu par le point de vue du Tractatus logico-philosophicus, puisqu'il ne peut y être question de l'existence d'entités mathématiques qui validerait nos énoncés à leur propos dans le sens de (1) sans que nous puissions les construire par nos calculs. Ce faisant, Wittgenstein doit irrémédiablement se ranger du côté des constructivistes ${ }^{28}$. Étant donné le caractère controversé de cette remarque, je ne peux pas la défendre ici. Je me contenterai donc de l'illustrer. En collaboration avec Paolo Mancosu, j'ai montré que Wittgenstein a donné dans ces manuscrits une nouvelle preuve mathématique, soit une version constructive de la preuve par Euler de l'infinité des nombres premiers, qui est un très bel exemple d'une preuve d'existence ${ }^{29}$. Cette version constructive n'a en fait rien de particulièrement remarquable, et nous n'avions pas l'intention de faire état de cette preuve dans le but de vanter les talents mathématiques de Wittgenstein, si ce n'est, minimalement, que pour montrer qu'il n'était pas aussi incompétent qu'on ne le croit d'ordinaire: nombre de ceux qui rejettent ces textes sous prétexte d'incompétence n'auraient pas fait mieux. L'intérêt de sa preuve tient plutôt dans le fait qu'il y transforme une preuve non constructive en une preuve constructive. S'il n'avait eu aucun intérêt envers ces questions, comme tant de ses interprètes le prétendent, pourquoi aurait-il pris la peine de faire cela? Pour illustrer le refus des preuves d'existence, je me contenterai simplement de reproduire ce qu'il en dit dans la section de la Grammaire philosophique consacrée à la preuve d'Euler. Après une brève description de celle-ci, il commente avec le style imagé qui lui est propre:

Nous nous trouvons ici de nouveau devant cette chose remarquable que l'on pourrait nommer «preuve mathématique par indice» — ce qui a toujours été

28. En m'appuyant sur une moyenne d'une demi-douzaine de citations par page, j'ai donné tout au long de (Marion, 1998) des raisons de croire que la position que prônait Wittgenstein est à rapprocher de ce qu'on entend ordinairement par "finitisme », sans pour autant entendre par là que nous devrions utiliser une définition du terme "finitisme» comme celle utilisée pour parler du programme de Hilbert, afin de caractériser la position de Wittgenstein (ce n'est pas la même chose), ce que les textes plus récents (Mancosu \& Marion, 2003) et (Marion \& Okada, 2011) ne font que confirmer. J'ai cependant pris la peine, dans Marion (2003 et 2008), de tirer au clair les liens entre cette position et l'intuitionnisme de Brouwer.

29. Voir Mancosu \& Marion, 2003. 
proscrit. Ou bien une preuve par symptômes. Le lien du symptôme avec ce que l'on voudrait avoir prouvé est lâche. Aucun pont n'a été construit, et voir l'autre rive nous suffit.

$[\ldots]$

Le pont doit être construit.

En mathématiques, il n'y a pas de symptômes; pour le mathématicien il ne peut y en avoir que dans un sens psychologique ${ }^{30}$.

\section{La notion de preuve-comme-trace et son caractère synoptique}

Dans la période de transition, Wittgenstein a élargi son point de vue, en proposant d'adjoindre des «matrices» comme celle-ci :

$$
\left|\begin{array}{ll}
a+(1+1) & =(a+1)+1 \\
a+(\xi+1) & =(a+\xi)+1 \\
a+((\xi+1)+1) & =(a+(\xi+1))+1
\end{array}\right|
$$

pour :

$$
a+(b+1)=(a+b)+1
$$

Ces matrices ont pour domaine la série $|1, \xi, \xi+1|$, qui n'est qu'une variante de la variable du 6.03 ci-dessus, pour générer de nouveaux calculs numériques $^{31}$. Elles présupposent dans le passage de la deuxième à la troisième ligne une preuve par induction mathématique, ce qui montre qu'il cherche désormais à élargir son point de vue pour rendre compte de ces dernières et qu'il commence à parler de preuves; c'est d'ailleurs en réfléchissant sur la notion d'induction propre à ces matrices qu'il a proposé ce qui est peut-être sa contribution la plus importante aux mathématiques, celle d'une règle d'unicité d'une fonction définie par récursion, en remplacement du principe d'induction mathématique ${ }^{32}$. Il faut bien comprendre que Wittgenstein voyait ces matrices comme établissant un lien entre l'algèbre (car la preuve qui permet de passer de la deuxième à la troisième ligne de la matrice ci-dessus est algébrique; c'est en modifiant la preuve de l'associativité de l'addition de Skolem que Wittgenstein à proposé sa règle d'unicité) et le langage des équations de la théorie des nombres, et que ce lien est pour lui direct et non dans le cadre d'un métalangage: il s'agit à la fois d'une différence fondamentale avec le programme finitiste de Hilbert ${ }^{33}$ et d'une

30. Wittgenstein, 1980, 390.

31. Wittgenstein, 1980, 426.

32. Wittgenstein, 1980, 403. Pour une discussion détaillée du raisonnement de Wittgenstein menant à cette règle d'unicité et l'usage qu'en fit son étudiant R. L. Goodstein, voir Marion \& Okada, 2011.

33. Voir Marion \& Okada, 2011. 
caractéristique propre à la philosophie de Wittgenstein, tout entière articulée autour de la distinction entre «dire» et «montrer», avec un refus concomitant de toute forme de métalangage.

L'argument dont je vais parler dans ce qui suit est en quelque sorte de la même nature, puisque Wittgenstein y insiste de nouveau sur le fait que le langage des équations de la théorie des nombres est indépendant du langage de la logique, même s'il veut bien reconnaître au moins ceci dans ses cours de 1939:

Les explications de Russell mettent en évidence, par exemple, le rapport de l'addition de deux nombres à la disjonction de deux concepts. $" 2+3=5$ » ne veut pas dire que vous mettez 2 ici et 3 là..., mais que si un concept possède le nombre 2 , un autre le nombre 3 , le concept qui représente la disjonction des deux précédents possède le nombre 5. [...] Ce que Russell et Frege ont fait est de relier les mots anglais et allemands "tous", "ou », «et », etc., aux énoncés numériques (numerical statements). Cela clarifie certaines choses (Wittgenstein 1995, 279-280).

Le lien entre les deux langages dont il parle ici est celui qu'on peut voir entre une équation comme:

(10) $27+16=43$

Et sa contrepartie dans le langage des Principia Mathematica ${ }^{34}$ :

(11) $\left(\exists !{ }_{27} x F(x) \wedge \exists !{ }_{16} x G(x) \wedge \forall x \neg(F(x) \wedge G(x))\right) \rightarrow\left(\exists / !{ }_{43} x(F(x) \vee G(x))\right)$.

Cette formule se lit comme suit:

S'il y a $27 F$ et $16 \mathrm{G}$, et si aucun $F$ n'est un $G$, alors il y a 43 choses qui sont ou bien $\operatorname{des} F$, ou bien des $G$.

Wittgenstein reconnaissait donc volontiers l'importance de ce lien entre les deux langages, parce qu'il permet certaines clarifications conceptuelles, qui se montrent directement, sans appel à un métalangage; ce sont des clarifications du type même dont il pensait que la philosophie devait être faite. Cependant, il pensait en outre pouvoir montrer avec des exemples de ce genre que les ambitions fondationnelles de Russell n'étaient pas justifiées. Comme il le disait déjà dans la Grammaire philosophique:

Ce n'est pas l'examen des concepts mais la seule compréhension du calcul numérique, qui peut nous dire que $3+2=5$. C'est ce qui en nous s'insurge contre l'idée que

«( $\exists 3 \mathrm{x}) . \varphi x .(\exists 2 \mathrm{x}) \cdot \psi \mathrm{x}$. Ind.: $\supset(\exists 5 \mathrm{x}) . \varphi \mathrm{x} \vee \psi \mathrm{x} »$

34. En fait, il n'existe pas de telle contrepartie dans Principia Mathematica, on peut dire tout au plus que la formule (11) est dérivée de l'assertion que « $1+1=2$ » au *54.43. Sur cette question voir (Marion, 2011). 
pourrait être la proposition $3+2=5$. Car ce qui nous fait reconnaître cette expression comme une tautologie ne peut soi-même résulter d'une réflexion sur les concepts, mais doit se montrer dans le calcul. Car la grammaire est un calcul ${ }^{35}$.

J'aimerais maintenant examiner l'argument de Wittgenstein à cet effet $^{36}$, dont l'occurrence la plus ancienne que je connaissance date d'une conversation avec Schlick et Waismann à Vienne en 192937, mais qui a joué par la suite un rôle prépondérant dans les écrits et les cours de 1938-193938, argument dont le but est de démontrer qu'une équation de la théorie des nombres n'est pas vraie en vertu du fait que la formule qui lui correspond dans le langage des Principia Mathematica soit une tautologie. C'est dans le cadre de ces remarques de Wittgenstein, qu'on trouve les raisons de croire que Wittgenstein s'en était tenu à une conception assez élémentaire de la preuve-comme-trace.

Appelons $N$ l'équation (10) et $R$ son correspondant russellien (11). L'argument de Wittgenstein peut être présenté de manière succincte si on reconnaît que les ambitions fondationnelles de Russell revenaient à ceci :

(12) N, N parce que $R$.

Il s'agit ici d'une forme "d'explicativisme » tirée en droite ligne de la distinction entre l'explication du fait ou de la réalité (o $\tau$ ) et l'explication du pourquoi $(\delta 10 \tau \imath)$ de cette réalité, faite par Aristote dans les Seconds analytiques, Livre I, section 13. Pour comprendre cette dernière, il suffit de considérer deux syllogismes Barbara:

Tous les $A$ sont $B$

Tous les $B$ sont $C$

$\therefore$ Tous les $A$ sont $C$

Pour Aristote, la proximité des planètes est établie par le fait qu'elles ne scintillent pas et le fait, établi indépendamment, que ce qui est proche ne scintille pas:

Les planètes ne scintillent pas.

35. Wittgenstein, 1980, 352. La formule « $(\exists 3 \mathrm{x}) \cdot \varphi \mathrm{x} .(\exists 2 \mathrm{x}) \cdot \psi \mathrm{x}$. Ind. : $\supset(\exists 5 \mathrm{x}) . \varphi \mathrm{x} \vee \psi \mathrm{x} »$ est inexacte, mais on peut la remplacer par l'équivalent de (11) pour « $3+2=5$ ».

36. J'ai déjà présenté ma lecture de cet argument dans Marion (2009) et discuté celle-ci de façon détaillée dans Marion (2011).

37. Voir Wittgenstein $(1991,45)$.

38. Voir en particulier la troisième partie des Remarques sur les fondements des mathématiques (Wittgenstein, 1983) et le cours XXVII dans Cours sur les fondements des mathématiques. Cambridge 1939 (Wittgenstein, 1995) où Wittgenstein discute de cet argument avec Alan Turing. On notera que, si Wittgenstein était en possession de l'argument dès 1929, ces textes et cours, où il le discute beaucoup plus en détail, datent de 1939. Il est fort probable que ce sont les objections de Turing qui forcèrent Wittgenstein à reconsidérer son argument. 
Tout ce qui est proche ne scintille pas.

$\therefore$ Les planètes sont proches.

Pour Aristote cela n'établit que le fait, puisque ce n'est pas parce qu'elles ne scintillent pas que les planètes sont proches. Pour une explication du pourquoi $(\delta \imath \tau \imath)$ il s'agit simplement d'inverser les termes $B$ et $C$ :

Les planètes sont proches.

Tout ce qui est proche ne scintille pas.

$\therefore$ Les planètes ne scintillent pas.

On a donc une explication qui donne la raison pour laquelle les planètes ne scintillent pas. Il faut cependant noter que nous avons deux syllogismes avec conclusions différentes et que le fait que les planètes sont proches n'est connu que par la conséquence de leur proximité, c'est-à-dire par le fait qu'elles ne scintillent pas $^{39}$. (Dans un cas empirique comme celuici, cela importe peu, puisque nous pouvons trouver d'autres manières de démontrer la proximité des planètes.)

Wittgenstein ne réfère pas à Aristote, mais il relève un défaut similaire pour (12): ce n'est pas parce que (11) est une tautologie que (10), mais c'est plutôt parce que nous connaissons déjà (10) que nous savons que (11) est une tautologie, donc Russell ne serait pas justifié d'affirmer (12). La raison en est que les indices numériques dans la formule (11) ne sont que des abréviations pour une formule contenant le nombre approprié de variables, soit 43 de chaque côté du conditionnel. Et on ne peut pas voir que ce nombre est le même de chaque côté, il faut compter les variables puis faire l'addition, ce qui revient à appliquer (10); on ne peut donc parler comme dans (12) de (11) comme donnant la garantie fondationnelle de la véracité de (10).

C'est dans le cadre de cet argument, dont je viens de brosser les grandes lignes, que l'on trouve les remarques de Wittgenstein montrant qu'il pense bien en termes de preuve-comme-trace. En effet, Wittgenstein insiste sur le fait qu'une preuve doit être "synoptique", car elle perdrait autrement son pouvoir de convaincre, donc son caractère même de preuve:

Je veux dire: si en modifiant sa notation on rend synoptique une figure de preuve qui ne l'était pas [nicht übersehbare Beweisfigur], on crée une preuve là où il n'en existait pas ${ }^{40}$.

Ce que Wittgenstein appelle "Beweisfigur», traduit ici par «figure de preuve" au sens géométrique du mot "figure", correspond à notre notion de «preuve-comme-trace». En effet, cette "figure de preuve» est une trace qui sert à refaire pour soi-même la preuve. Il n'y a donc pas de preuve là où

39. Voir Dubucs et Lapointe (2003, 222-223).

40. Wittgenstein, 1983, III, $\mathbb{} 2$. 
la «figure de preuve " n'est pas «synoptique ». Et le caractère «synoptique» ne se réduit pas simplement à la capacité qu'a cette "figure de preuve » d'être recopiée aisément ${ }^{41}$, puisqu'on peut recopier toutes les étapes d'une preuve sans pour autant comprendre en quoi elles sont collectivement nécessaires et suffisantes pour prouver le théorème. Wittgenstein parle aussi à l'occasion de la Beweisfigur comme devant être une "einprägsames Bild» ou «image marquante » ${ }^{42}$, l'idée étant en quelque sorte qu'elle doit posséder une structure dont l'appréhension et la remémoration soit suffisamment aisée pour que le mathématicien puisse reproduire la preuve au besoin.

Wittgenstein peut donc rejeter l'argument selon lequel on peut se contenter de la formule abrégée (11) comme fondement dans (12). Moyennant l'identification des variables, dans la version non abrégée de (11), à des bâtonnets, son argument repose donc sur l'idée que dans:

(13) ||||||||||||||||||

on ne peut pas savoir par une simple inspection visuelle, donc sans compter, s'il y a 15 ou 16 bâtonnets. En effet, il écrivait déjà en 1929:

Comment puis-je savoir que IIIIIIII est le même signe que IIIIIIIII ? Il ne suffit pourtant pas qu'ils se ressemblent. Car l'identité des signes ne consiste pas en l'identité approximative des formes [Gestalt] mais précisément dans l'identité des nombres ${ }^{43}$.

Puisque que la formule (11), lorsqu'elle n'est plus abrégée, perd en tant que "figure" son caractère "synoptique ", elle ne peut donc plus servir de «fondement» au sens de (12) ${ }^{44}$. Dans ses carnets, le 18 novembre 1939 (au MS 122, 28r), Wittgenstein dessine:

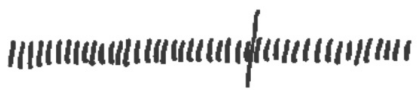

41. Ce sens de «synoptique» est introduit dans Wittgenstein (1983, III, $\mathbb{~ 1 ) ~}$

42. Par exemple, Wittgenstein (1983, III, $\mathbb{9}$ 9).

43. Cette citation est tirée de la Grammaire philosophique de 1933 (Wittgenstein, 1980, 336-337), mais l'origine de ce passage est dans le MS 105 de 1929, et il apparaît aussi dans les Remarques philosophiques (Wittgenstein, 1975, $\mathbb{S} 103$ ). Cet exemple montre bien que la réflexion de Wittgenstein sur ces questions est bien plus ancienne qu'on ne le croit d'ordinaire, et que l'image d'une transition durant les années 1929-1935 vers une philosophie tardive, le « deuxième Wittgenstein », qui ne pourrait guère être assimilé à ses écrits de transition, est largement basée sur l'ignorance des textes.

44. On pourrait objecter qu'il est possible, par exemple, de joindre les variables une à une et ne voir que le nombre, le même de chaque côté du conditionnel, mais Wittgenstein pourrait alors rétorquer que cette procédure ne devient plus fiable avec de très grand nombres. Voir, par exemple Wittgenstein (1983, III, $\$$ 6-7). D'autres objections sont possibles, qui sont discutées dans Marion (1998, 228sq., et 2011) et Mühlhölzer (2005). 


\section{Et commente:}

Cette figure est-elle une preuve de $27+16=43$ parce qu'on arrive à « 27 » en comptant les traits du côté gauche et à « 16 » quand on compte ceux du côté droit, et à « 43 » quand on compte la série entière?

En quoi est-ce étrange que nous appelions cette figure une preuve de cette proposition? Cela tient à la façon dont il faut reproduire et reconnaître [wiederzuerkennen] cette preuve; en ce qu'elle n'a pas de forme visuelle caractéristique [daß er keine charakteristische visuelle Gestalt hat].

Même si la preuve n'a aucune forme visuelle de ce genre, cela ne m'empêche pas de la copier (de la reproduire) exactement - cette figure n'est-elle donc pas encore une preuve? Je pourrais par exemple la graver dans l'acier et la faire passer de main en main. Je dirais alors à un tiers: "Vous avez là la preuve de $27+16=43$ » - Ne peut-on pas dire malgré tout qu'elle prouve la proposition à l'aide du modèle? Certes, mais le modèle n'est pas la preuve [die Figur ist nicht der Beweis $]^{45}$.

Je crois qu'il n'est pas utile de multiplier les passages à l'appui, les lecteurs de la troisième partie des Remarques sur les fondements des mathématiques auront reconnu ce dont je parle. Pour reprendre les distinctions (i)-(iii) de départ, on voit très bien que Wittgenstein distingue ici Beweis et Beweisfigur ou simplement Figur et que cette dernière sert de trace objective (ii) - gravée ici dans l'acier! - pour que chaque mathématicien puisse effectuer à son tour à partir d'elle la preuve comme acte subjectif (i), et que c'est pour cette raison que Wittgenstein réclame que la Beweisfigur soit «synoptique» [übersehbar $]^{46}$. Elle ne pourrait pas remplir son rôle autrement; l'argument de Wittgenstein étant que le dessin ci-dessus ne peut justement pas remplir ce rôle, ce qui en soi n'a rien de surprenant, mais implique cependant en contrepartie que la version non abrégée de (11) ne peut pas remplir le rôle que lui fait jouer (12).

\section{Conclusion}

Je n'ai pas cherché dans ce qui précède à évaluer l'argument de Wittgenstein, mais simplement à l'utiliser pour mettre en lumière sa compréhension de (2). Il me suffit donc pour conclure de rappeler ce qui a été démontré: j’ai montré en premier lieu comment Wittgenstein excluait les mathématiques (en fait, la théorie des nombres) du domaine de (1), et qu'il partageait donc (2), par défaut, avec les intuitionnistes. Cependant, la notion de preuve à l'œuvre dans (2) selon l'intuitionnisme est celle de la preuve-comme-objet, et je viens de montrer que Wittgenstein n'avait en tête qu'une conception de la preuve-

45. Wittgenstein, 1983, III, $\mathbb{} 11$.

46. Depuis Church $(1956,52)$, on reconnait que les preuves mathématiques doivent être «effectives» parce qu'elles doivent être communicables. Le critère que propose ici Wittgenstein pour la preuve-comme-trace est beaucoup plus fort. 
comme-trace. Donc, si Wittgenstein partage (2) avec les intuitionnistes, le sens qu'il donne à cette analyse est différent.

On pourrait reprocher à ma présentation le fait que j'ai utilisé pour la première partie de mon argument des remarques tirées du Tractatus logicophilosophicus, tandis que dans la deuxième partie de l'argument j'ai utilisé des remarques tardives, comme cette dernière citation datant de 1939, et que j'ai ainsi fait fi de l'évolution de la pensée de Wittgenstein. Certes, beaucoup de détails changent au cours des ans, mais je crois que l'idée d'une évolution vers un "deuxième Wittgenstein" est trop souvent exagérée et parfois utilisée pour couvrir l'incapacité que l'on a à donner une autre lecture des remarques en question. De surcroît, Wittgenstein est encore de nos jours beaucoup trop souvent lu à rebours, en commençant par une interprétation hors contexte de ce "deuxième Wittgenstein", pour le relire dans le "premier Wittgenstein », c'est-à-dire dans le Tractatus logico-philosophicus, en ignorant souvent ce qu'on appelle la "période de transition", ces textes finalement méprisés de 1929-1935. Je crois, contre ce genre de contresens exégétique, qu'il faut d'abord comprendre le Tractatus logico-philosophicus dans son contexte, puis montrer, à travers la lecture des écrits de la "période de transition", comment Wittgenstein a évolué vers ses dernières positions. En fait, je n'ai rien fait ici qui ne respecte ce principe et je crois au contraire que les remarques plus tardives deviennent beaucoup plus transparentes lorsqu'on a compris ce que Wittgenstein a voulu faire dans son Tractatus logico-philosophicus et, par la suite, les changements qu'il a apportés aux positions défendues dans ce livre. L'argument contre Russell sur le caractère synoptique des preuves s'éclaire donc du point de vue du Tractatus logicophilosophicus, c'est-à-dire du point de vue de la séparation que Wittgenstein fait entre le langage des équations de la théorie des nombres et le langage de la logique. Par ailleurs, la réflexion sur l'analyse de la notion de vérité en termes de "vérifacteurs", elle aussi tirée du Tractatus logico-philosophicus et une réflexion sur l'obligation, à laquelle Wittgenstein fit face en soustrayant les mathématiques du domaine de (1), d'adopter (2), a aussi été d'une très grande utilité pour éclairer ces remarques tardives, dans la mesure où on peut ainsi mettre en relief la distinction entre Beweis et Beweisfigur, qui est essentielle pour comprendre un des arguments les plus importants des Remarques sur les fondements des mathématiques de Wittgenstein, sur le caractère synoptique de la preuve.

\section{Bibliographie}

Beall, J. C. \& G. Restall. Logical Pluralism, Oxford, Oxford University Press, 2006. Beebee, H. \& Dodd, J. (ed.). Truthmakers: The Contemporary Debate, Oxford, Oxford University Press, 2005.

Brouwer, L. E. J. Collected Works. Volume 1: Philosophy and the Foundations of Mathematics, A. Heyting (ed.), Amsterdam, North-Holland, 1976. 
- «Les principes logiques ne sont pas sûrs », dans F. Rivenc \& P. de Rouilhan (ed.), Logique et fondements des mathématiques (1850 - 1914), Paris, Payot, 1992, 386-392.

Church, A. Introduction to Mathematical Logic, Princeton, Princeton University Press, 1956.

Diamond, C. L'esprit réaliste. Wittgenstein, la philosophie et l'esprit, Paris, Presses Universitaire de France, 2004.

Dubucs, J. et S. Lapointe. «Preuves par excellence», Philosophiques, vol. 30, 2003, 219-234.

Dummett, Sir Michael A. E. The Seas of Language, Oxford, Clarendon Press, 1993.

- Elements of Intuitionism, $2^{\mathrm{e}}$ éd., Oxford, Clarendon Press, 2000.

Frascolla, P. Wittgenstein's Philosophy of Mathematics, Londres, Routledge, 1994.

—. "The Tractatus System of Arithmetic», Synthese, vol. 112, 1997, 353-378.

Granger, G.-G. "Wittgenstein et la métalangue», dans Invitation à la lecture de Wittgenstein, Aix-en-Provence, Alinéa, 1990, 159-171.

Heyting, A. "Sur la logique intuitionniste», Académie royale de Belgique. Bulletin de la classe des sciences, vol. 16, 1930, 957-963.

—. «Die intuitionistische Grundlegung der Mathematik», Erkenntnis, vol. 2, 1931, 106-115.

Kolmogorov, N. "Zur Deutung der intuitionistischen Logik», Mathematische Zeitschrift, vol. 35, 1932, 58-65.

Lowe, E. J. \& A. Rami (dir.). Truth and Truth-Making, Montreal/Kingston, McGillQueen's University Press, 2009.

Mancosu, P. et M. Marion. «Wittgenstein's Constructivization of Euler's Proof of the Infinity of Prime Numbers ", dans F. Stadler (dir.), The Vienna Circle and Logical Empiricism: Re-evaluation and Future Perspectives, Dordrecht, Kluwer, 2003, 171-188.

Marion, M. Wittgenstein, Finitism and the Foundations of Mathematics, Oxford, Clarendon Press, 1998.

-. "Qu'est-ce que l'inférence? Une relecture du Tractatus logico-philosophicus », Archives de philosophie, vol. 64, 2001, 545-567.

_. "Wittgenstein and Brouwer», Synthese, vol. 137, 2003, 103-127.

- "Brouwer on Hypotheses and the Middle Wittgenstein ", dans M. van Atten, P. Boldini, M. Bourdeau \& G. Heinzmann (dir.), One Hundred Years of Intuitionism (1907-2007). The Cerisy Conference, Bâle, Birkhäuser, 2008, 96-114.

—. "Radical Anti-Realism, Wittgenstein and the Length of Proofs ", Synthese, vol. 171, 2009, 419-432.

—. "Wittgenstein on the Surveyability of Proofs ", dans M. McGinn \& O. Kuusela (dir.), The Oxford Handbook on Wittgenstein, Oxford, Clarendon Press, 2011, à paraître.

Marion, M. \& M. Okada. "Wittgenstein and Goodstein on the Equation Calculus and the Uniqueness Rule», 2011, à paraître.

Monnoyer, J.-M. (dir.). Metaphysics and Truth-Makers, Francfort, Ontos Verlag, 2007.

Mühlhölzer, F. «"A Mathematical Proof Must be Surveyable” What Wittgenstein Meant by This and What it Implies ", Grazer Philosophische Studien, vol. 71, 2005, 57-86. 
Mulligan, K. "Truth and the Truth-Maker Principle in 1921 », dans Lowe \& Rami (2009), 2009, 39-58.

Mulligan, K., P. Simons \& B. Smith. "Truth-Makers ", Philosophy and Phenomenological Research, vol. 44, 1984, 287-321.

Potter, M. Reason's Nearest Kin, Oxford, Clarendon Press, 2000.

Quine, W. v. "Grammar, Truth, and Logic ", in S. Kanger \& S. Öhman (ed.), Philosophy and Grammar, Dordrecht, D. Reidel, 1980, 17-28.

- Philosophy of Logic, $2^{\mathrm{e}}$ édition, Cambridge Mass., Harvard University Press, 1986.

Sundholm, G. "Constructions, Proofs and the Meaning of Logical Constants ", Journal of Philosophical Logic, vol. 12, 1983, 151-172.

—. "Questions of Proof », Manuscrito, vol. 16, 1993, 47-70.

- «Existence, Proof, and Truth-Making: A Perspective on the Intuitionistic Conception of Truth». Topoi, vol. 13, 1994a, 117-126.

—. "Proof-Theoretical Semantics and the Fregean Identity Criteria for Propositions ", The Monist, vol. 77, 1994b, 294-314.

- - "Implicit Epistemic Aspects of Constructive Logic ", Journal of Logic, Language, and Information, vol. 6, 1997, 191-212.

—. "Inference, Consequence, Implication. A Constructivist Perspective », Philosophia Mathematica (3), vol. 6, 1998a, 178-194.

—. "Inference vs. Consequence », in T. Childers (dir.), The Logica Yearbook 1997, Prague, Filosofia, The Institute of Philosophy, Academy of Sciences of the Czech Republic, 1998b, 26-35.

Tarski, A. "Sur le concept de conséquence logique», dans Logique, sémantique et métamathématique 1923-1944, vol. 2, Paris, Armand Colin, 1974, 141-152.

—. "What are Logical Notions?", History and Philosophy of Logic, vol. 7, 1986, 143-154.

Wittgenstein, L. Remarques philosophiques, Paris, Gallimard, 1975.

—. Grammaire philosophique, Paris, Gallimard, 1980.

- Remarques sur les fondements des mathématiques, Paris, Gallimard, 1983.

- Wittgenstein et le Cercle de Vienne. D'après les notes de Friedrich Waismann, Mauvezin, T.E.R, 1991.

—. Tractatus logico-philosophicus, Paris, Gallimard, 1993.

. Cours sur les fondements des mathématiques. Cambridge 1939, Mauvezin, T.E.R, 1995. 